\title{
Las nuevas atribuciones del INE al interior de las elecciones locales
}

\section{Arturo Sánchez Gutiérrez* Horacio Vives Segl**}

\author{
Sumario: \\ I. Planteamiento \\ II. La reforma: un paso necesario \\ III. El INE y los OPLE: una relación de colaboración institucional \\ IV. Tres opciones de coordinación: asunción, atracción \\ y delegación \\ V. Asunción \\ VI. Atracción \\ VII. Delegación
}

* Consejero electoral ante el Consejo General del Instituto Nacional Electoral.

** Catedrático en el Departamento de Ciencia Política del Instituto Tecnológico Autónomo de México e investigador asociado de El Colegio de México.

Los autores agradecen a Eduardo Nateras Martínez la revisión de las primeras versiones del texto. 


\section{Planteamiento}

El presente artículo tiene como propósito hacer una reflexión sobre la manera en que la reforma constitucional en materia político electoral de 2013-2014 y la reforma legal de 2014 derivada de la anterior, impactaron en la naturaleza de los órganos electorales —-tanto a nivel federal como estatal- y el nuevo modelo de organización electoral que se produce a partir de entonces. En razón de lo anterior, el artículo se dividirá en dos partes. En la primera de ellas, se hará un repaso de los principales elementos de la discusión, previos a la reforma constitucional. En la segunda, se detallará y reflexionará sobre las alternativas que se abren tras la promulgación del nuevo ordenamiento legal.

\section{La reforma: un paso necesario}

El inicio del gobierno del presidente Enrique Peña Nieto se caracterizó por la firma de un acuerdo político que entonces se anunciaba como una agenda de transformaciones profundas para el país. Dicho acuerdo era el "Pacto por México", suscrito por el gobierno federal y las tres principales fuerzas políticas - el Partido Revolucionario Institucional (PRI), el Partido Acción Nacional (PAN) y el Partido de la Revolución Democrática (PRD) -.${ }^{1}$ El documento constaba de diversos apartados. El quinto de ellos, se refería a los acuerdos a adoptar en materia de "gobernabilidad democrática”. Los compromisos 89 y 90 marcaban una agenda mínima para discutir contenidos de la futura reforma electoral. Puntualmente, uno de los apartados del compromiso 90 establecía a la letra: "Crear una autoridad electoral de carácter nacional y una legislación única, que se encargue tanto de las elecciones federales, como de las estatales y municipales". Fue así que los primeros meses de 2013 estuvieron marcados por diversas discusiones sobre los alcances que tendría la reforma electoral

1 El Pacto por México fue firmado el 2 de diciembre de 2012 en el Castillo de Chapultepec. Por el gobierno federal, firmó Enrique Peña Nieto, presidente de la República, Jesús Zambrano Grijalva, presidente del PRD, Gustavo Madero Muñoz, presidente del PAN y María Cristina Díaz Salazar, presidenta del PRI. Véase www. pactopormexico.org/ PACTO-POR-MEXICO-25.pdf (acceso: 28 de abril de 2014). 
y la autoridad electoral de carácter nacional, establecidos en el compromiso 90.

Con el correr de las semanas del primer semestre de 2013, empezaron a sumarse voces críticas a la reforma electoral. El motivo era evitar - al menos en un segmento de la opinión pública, capaz de generar una resistencia articulada - la creación de una autoridad electoral única centralizada, que afectara al federalismo electoral ya consolidado en los estados - mal que bien-. Se polemizaba sobre la ocurrencia misma de la reforma electoral, bajo el argumento de que el Instituto Federal Electoral (IFE), ya había adquirido prestigio y que no era necesario meterse a innovar en la celebración de elecciones cuando ya se habían obtenido buenos resultados. En particular resultaba cuestionable afectar el ámbito de competencia de cada autoridad electoral (federal y local). Además, se criticaba que de nueva cuenta, una vez ocurridas elecciones presidenciales, se modificara la estructura electoral. ${ }^{2}$ Si bien la elección presidencial de 2012 tuvo ciertos rasgos polémicos y fue cuestionada por la izquierda, ${ }^{3}$ las inconformidades fueron superadas relativamente pronto, sin los conflictos poselectorales que caracterizaron, por ejemplo, a las elecciones presidenciales de 1988 o de 2006. Los organismos estatales electorales cerraban filas para tratar de combatir iniciativas que se tradujeran en una disminución en su autonomía y facultades, o - en el más extremo de los casos- que eliminara su existencia misma. Todas esas críticas y circunstancias se abroquelaban en un frente común para resistir el avance de la reforma. Con todo, la voluntad de los partidos políticos y del gobierno federal iba avanzando - no sin dificultades-, fruto del "pactismo" para acordar los contenidos de la reforma electoral.

Algo que estuvo presente durante la época de discusión de la reforma política, es que ésta no lograba avanzar por sus propios méritos, sino que estaba atada a la suerte de otras reformas que se derivaban de la firma del Pacto por México. La primera de las reformas constitucionales desde el

2 Cabe señalar que, con excepción de la elección presidencial de 2000, que dio lugar a la primera alternancia en la Presidencia de la República, desde 1976 después de cada elección presidencial, se detonaba algún conflicto poselectoral que buscaba solucionarse con reformas electorales.

3 Una revisión crítica de las elecciones de 2012 se encuentra en mi artículo en autoría conjunta con Horacio Vives Segl, "El desempeño de la actividad electoral: organización y arbitrariedad con una nueva ley", en Alvarado, Arturo (comp.), Las elecciones en México, México, Centro de Estudios Sociológicos-El Colegio de México-Instituto Nacional Electoral (en prensa). El 11 de noviembre de 2013 se celebró un coloquio en El Colegio de México en el que se presentó el artículo referido. 
inicio de la administración de Peña Nieto — la educativa一, se logró en enero de 2013 y sin mayores controversias (si bien, como sería evidente, la negociación de las reformas secundarias se daría en un marco de fuerte complejidad, que incluso llevaría a manifestaciones que obligaron a los legisladores a sesionar en una sede alterna). Pero a partir de entonces, las reformas llamadas "de gran calado", eran percibidas como piezas de cambio en un tablero de negociaciones, de tal manera que las discusiones de la reformas energética, política, telecomunicaciones y hacendaria estuvieron relacionadas entre sí, más allá de las especificidades de la propia reforma político electoral.

Otro obstáculo que se presentó en aquel momento, fue la denuncia que hizo el presidente del Partido Acción Nacional sobre el presunto uso de recursos públicos de programas sociales para beneficiar campañas electorales de candidatos vinculados con gobernantes en turno del PRI, específicamente en Veracruz. Dichas denuncias fueron motivo de una adenda, para garantizar la limpieza y erradicar y sancionar conductas ilegales de las elecciones locales que estaban en curso en ese momento en 15 estados de la República y cuya jornada electoral habría de celebrarse el 7 de julio de 2013. Paradójicamente, las irregularidades que se presentaron en la organización del proceso electoral en algunos estados - particularmente en Baja California (única entidad en la que estuvo en juego la gubernatura) y en Tlaxcala - fueron reafirmando la convicción de que la ausencia de procedimientos homologados para la realización de elecciones locales, era un problema cada vez más evidente que se podría solucionar con una legislación y reglamentación única. Aunado a ello, la intromisión cada vez más publicitada de los gobernadores en los supuestos órganos electorales locales autónomos, también abonó en la necesidad de limitar su injerencia sobre las autoridades electorales locales. ${ }^{4}$ Los meses del segundo semestre de 2013 se caracterizaron por los debates, tanto en el ámbito legislativo, como en foros y opinión publicada, sobre la agenda de una reforma político electoral que ya era percibida como irreversible.

4 Una reciente fuente de documentación de la indebida injerencia de los gobernadores en los procesos electorales locales se encuentra en el estudio de Irma Méndez de Hoyos, "Los órganos de administración electoral y la calidad de los institutos electorales estatales", en Méndez de Hoyos, Irma y Loza Otero, Nicolás (coords.), Instituciones electorales, opinión pública y poderes políticos locales en México, México, Flacso-México, 2013, pp. 27-58. 
Fue así que hacia finales del año se logró el acuerdo que dotó de contenidos a la reforma político electoral. ${ }^{5}$ El punto principal de la reforma, fue la creación del Instituto Nacional Electoral (INE) que sustituiría al IFE como máxima autoridad electoral administrativa en el país, con una reasignación de facultades (de eso se hablará más adelante). Un primer aspecto que merece una mención, es que la reforma constitucional no solo detalló aspectos sobre la organización de las elecciones y la naturaleza de las autoridades electorales. La reforma contempló aspectos que en el mediano plazo, están pensados para mejorar el funcionamiento de algunas de las instituciones de nuestra democracia. ¿Cuáles son las virtudes de la reforma política en rango constitucional aprobada en diciembre de 2013 y promulgada en febrero de 2014, más allá de los cambios sobre la naturaleza y funciones de las autoridades electorales?

- Termina la prohibición histórica - y con ello su eterno amateurismo y limitada rendición de cuentas- que impedía la reelección consecutiva de legisladores federales y locales y de las autoridades municipales.

- Los senadores y diputados federales se podrán reelegir consecutivamente hasta por doce años (cuatro periodos de legislaturas trianuales para diputados y dos periodos de legislaturas sexenales para senadores) a partir de 2018 .

- Los diputados locales se podrán reelegir hasta por cuatro periodos y las autoridades municipales hasta por dos (en tanto la administración no dure más de tres años).

- Conversión en 2018 de la actual Procuraduría General de la República en una Fiscalía General, con dos áreas especializadas (combate a la corrupción y delitos electorales), cuyo nombramiento y remoción del titular ya no dependerá exclusivamente del presidente de la República, sino que deberá contar con la aprobación de dos terceras partes del Senado.

- Establecimiento de un máximo de $8 \%$ de diferencia entre la votación obtenida y la representación proporcional asignada a los partidos políticos en los congresos locales.

5 La reforma político-electoral fue aprobada el 13 de diciembre de 2013 por el Congreso de la Unión. Como se sabe, a partir de ahí tuvo que pasar por la aprobación de por lo menos la mitad más uno de los congresos locales para continuar con los requisitos legales. El 22 de enero de 2014 se emitió la declaratoria de constitucionalidad al aprobarse por 18 legislaturas locales. Finalmente, el decreto fue promulgado y publicado en el Diario Oficial de la Federación el 10 de febrero de 2014. 
- Paridad de género en la presentación de candidaturas a las cámaras de diputados y senadores del Congreso de la Unión.

- Posibilidad de formar gobiernos de coalición, que requerirían la aprobación del Senado (con excepción de los titulares de Marina y Defensa).

- Ratificación del secretario de Hacienda por parte de la Cámara de Diputados y del secretario de relaciones exteriores por parte del Senado.

- Ratificación por parte del Senado de la estrategia nacional de seguridad del presidente de la República, quien deberá rendir un informe anual de resultados.

- Cambio de toma de posesión del 1o. de diciembre al 1o. de octubre en la Presidencia de la República.

- Cambio en el inicio del primer periodo ordinario de sesiones del Congreso de la Unión del 1o. de septiembre al 1o. de agosto, cuando el presidente de la República inicie su gobierno.

- Autonomía a partir de 2018, del Consejo Nacional de Evaluación de la Política de Desarrollo Social (Coneval).

He ahí un apretado resumen de contenidos de la reforma política, más allá de los aspectos estrictamente electorales. Se criticó a la reforma -en todo o en algunas partes - sin establecer una justa valoración en el sentido de que no solo se trató de un cambio en la naturaleza de la autoridad electoral: la reforma política de 2013 lleva la semilla de un cambio en diversas instituciones públicas para continuar consolidando en el corto y mediano plazo a la democracia mexicana.

Por lo que respecta a las instituciones y procedimientos específicamente electorales - además de la creación del Instituto Nacional Electoral (INE), "la joya de la reforma"-, éstas quedaron contempladas en las siguientes novedades:

- Trasformación de los institutos estatales electorales, u órganos equivalentes, en organismos públicos locales en materia electoral, denominados OPLE.

- Unificación del órgano superior de dirección de los OPLE, con el nombramiento de seis consejeros electorales y un consejero presidente.

- Los consejeros electorales y el consejero presidente de los consejos locales de los OPLE serán nombrados y removidos por el Consejo General del INE. 
- Incremento del 2 al 3\% del porcentaje mínimo necesario para mantener el registro como partido político nacional; mismo umbral que deben alcanzar los partidos para tener acceso al reparto de diputados plurinominales.

- Jornada electoral federal que se adelanta del primer domingo de julio al primer domingo de junio.

- Creación del Servicio Profesional Electoral Nacional, que determinará las directrices en la selección, promoción, permanencia, rotación, evaluación y sanción de los servidores públicos tanto del INE, como de los OPLE.

- Nuevas causales de nulidad en las elecciones: rebasar en más de 5\% el monto de gastos de campaña permitido, la adquisición de tiempos en radio y televisión fuera de norma y el uso o recepción en campañas electorales de recursos públicos o de procedencia ilícita.

- Regla para la asignación de financiamiento a candidatos independientes; la suma de ellos será considerada como un solo partido político en el reparto del componente igualitario de financiamiento público.

Una vez especificados los grandes trazos de la reforma constitucional en materia político electoral, procederemos a discutir la parte correspondiente a la naturaleza de la nueva autoridad electoral de carácter nacional —el INE-, así como las funciones y la relación de los OPLE con el Instituto.

III. El INE y los OPLE: una relación de colaboración institucional

Conforme fue avanzando la discusión de la reforma política y se percibía como inminente la creación del INE, se fueron sucediendo diversos debates. Dichos debates iban desde la denominación misma de la nueva autoridad electoral nacional: si se debía mantener el nombre del Instituto Federal Electoral - en el entendido de que era una "marca" con reconocimiento y prestigio-, o si atendiendo a consideraciones jurídicas y la novedad política, tendría que darse una nueva denominación institucional. Fue así que tras casi 24 años de existencia, desapareció el Instituto Federal Electoral para dar lugar al Instituto Nacional Electoral.

Otro de los debates se refería a si el INE se tendría que hacer cargo de todas las elecciones - las federales que venía organizando (presidente 
de la República y diputados y senadores de mayoría relativa y de representación proporcional), más las locales (gobernadores, diputados locales y autoridades municipales, así como los equivalentes en el Distrito Federal) - o solo de algunas de las facultades que correspondían a los institutos estatales electorales. En suma, desparecer a los órganos locales o mantenerlos. Y en caso de permanecer, ¿cómo quedaría su autonomía y la distribución de facultades? ¿Qué tareas o procesos apoyaría el INE y cuáles serían responsabilidad de las autoridades locales? ${ }^{6}$

Como se sabe, se suscitaron fuertes resistencias y argumentos para evitar la desaparición de los institutos estatales electorales. Se presentaron argumentos concentrados en dos grandes grupos: por un lado, un supuesto "ataque al federalismo electoral" que implicaba "una regresión a la centralización" - argumento que no se sostiene, al ser justamente las legislaturas locales que participando del constituyente permanente, avalaron la reforma constitucional-, y por otro lado, que se iba a incrementar en gran medida el gasto electoral, derivado de las múltiples liquidaciones de personal, cuya experiencia en la materia, adicionalmente, no era tomado en cuenta. El fin de esa historia es conocido, al mantenerse la existencia de los organismos locales, pero bajo ciertas premisas contempladas en la Constitución.

Los institutos estatales electorales —algunos "de participación ciudadana"-, darían paso a los OPLE. Si bien se preservaba su existencia, autonomía y una naturaleza similar a los organismos que les precedieron, los nuevos OPLE tendrían que trabajar y definirse en función de una rectoría del INE, empezando por el hecho de que los integrantes del órgano superior de dirección a nivel local, con voz y voto en el Consejo, serían nombrados por el Consejo General del INE, lo cual abonaba a la sensación de cierta jerarquía del INE sobre los OPLE. Por otro lado, se establecían tres supuestos sobre la forma en que el INE podría participar en las elecciones locales - por medio de la atracción, la asunción o la

6 Solo a manera de ejemplo, abrimos aquí un paréntesis sobre el delicado tema del financiamiento y fiscalización de los recursos de los partidos políticos para ilustrar uno de los grandes temas que son responsabilidad de la autoridad electoral, del orden nacional o estatal. Una vez aprobada la reforma, el INE se enfrentaba a un dilema - no menor- sobre qué hacer en materia de fiscalización: centralizar o delegar en los órganos locales electorales tan relevantes tareas para una competencia equitativa entre los contendientes electorales. El INE decidió centralizar la fiscalización de las elecciones y establecer lineamientos y un sistema novedoso de contabilidad. Para abundar sobre los dilemas de la fiscalización de los partidos, véase Sánchez Gutiérrez, Arturo, "La lupa sobre el dinero", Voz y Voto, núm. 257, julio de 2014. 
delegación-, independientemente del artículo 116 constitucional, que preservó la autoridad y fuerza que se les daba a los OPLE para actuar a nivel estatal.

Esas eran las premisas básicas de la naturaleza y constitución de las autoridades locales. Tras quedar en vigencia la reforma constitucional, el paso siguiente era la aparición de la nueva ley secundaria que sustituiría al Cofipe, situación que tardaría unos meses, al ser sancionada a finales de mayo de 2014 la Ley General de Instituciones y Procedimientos Electorales (LGIPE). ${ }^{7}$ Quedarían entonces definidas las tres condiciones bajo las cuales el INE podría participar en las elecciones locales. Adicionalmente, la LGIPE estableció la creación de una Comisión de Vinculación - integrada por cuatro consejeros electorales - como instancia de coordinación entre la autoridad electoral nacional y sus homólogos locales, y se establecieron los lineamientos y la convocatoria para el proceso de selección - por parte del Consejo General del INE- de los aspirantes a ser designados consejeros electorales de los OPLE.

\section{Tres opciones de coordinación: asunción, atracción y delegación}

Desde el momento en que se llevó a cabo la reforma constitucional, se modificó el quehacer de la autoridad electoral. Entre las nuevas funciones del INE - además de la realización de elecciones federales que venía desempeñando el IFE desde su creación, y la administración de los tiempos del Estado en materia electoral (que viene desde la reforma de 2007) - se contempla la organización de consultas populares; a solicitud de los partidos y con cargo a sus prerrogativas; la organización de los procesos internos de elección de sus dirigentes; y la participación en diversas formas en la realización de los procesos electorales locales.

Antes de entrar al análisis de las nuevas atribuciones del INE, se debe tener en cuenta que la reciente legislación - tanto en la Constitución como en la LGIPE - establece las atribuciones del INE cuando se trate de elecciones únicamente federales; las funciones del INE para ambos tipos de elecciones - tanto federales como locales-, y las funciones y atribuciones de los OPLE en la organización de elecciones únicamente locales.

7 Inclusive, en el periodo que va de la entrada en vigencia de la reforma constitucional a la de la LGIPE, se nombraron, el 4 de abril de 2014, a los consejeros electorales y al consejero presidente del Consejo General del INE. 
Tras la publicación de la LGIPE en el Diario Oficial de la Federación, entraron en vigencia, entre otras cosas, tres nuevas formas de interacción entre el Instituto Nacional Electoral y los organismos públicos locales en materia electoral: la facultad de asunción, la de atracción y la de delegación. Estas nuevas atribuciones pueden llevarse a cabo durante la organización y desarrollo de procesos electorales únicamente locales.

Si bien la ley contempla que los OPLE desempeñen funciones específicas en organización de los procesos electorales en las respectivas entidades federativas, estas tres nuevas atribuciones dieron al instituto de carácter nacional, facultades por encima de las autoridades electorales locales para la organización de elecciones en dicho ámbito. Por tal motivo, los organismos locales deberán cumplir con determinados lineamientos emitidos por el INE, durante la organización y desarrollo de las elecciones en los estados.

\section{Asunción}

De acuerdo con lo establecido en la Constitución, en el desarrollo de elecciones locales, los OPLE estarán encargados de cumplir con las siguientes funciones:

- Determinar el derecho y acceso a las prerrogativas de partidos políticos y candidatos.

- Hacerse cargo de la educación cívica.

- Preparar la jornada electoral.

- Imprimir documentos y producir materiales electorales.

- Llevar a cabo el escrutinio y los cómputos, siguiendo determinadas condiciones.

- Declarar la validez de las elecciones locales y otorgar las constancias correspondientes.

- Realizar el cómputo de la elección del titular del Poder Ejecutivo.

- Emitir resultados preliminares, encuestas o sondeos de opinión; así como encargarse de la observación electoral y los conteos rápidos, de acuerdo con los lineamientos correspondientes.

- Encargarse de la organización, desarrollo, cómputo y declaración de resultados en los mecanismos de participación ciudadana que contemplen las leyes locales. 
Una vez establecidas las funciones de los OPLE, de acuerdo con la Legipe, la asunción queda entendida como una atribución del INE para asumir directamente la realización de todas las actividades electorales que les corresponden a dichos organismos locales. Sin embargo, esta atribución del Instituto no es de uso discrecional, y podrá ejercerse únicamente bajo condiciones específicas, y a petición fundada ante el Instituto de cuando menos cuatro consejeros electorales, o por parte de la mayoría de los integrantes del consejo del organismo público local en cuestión. En ambos casos, la petición deberá presentarse antes de que inicie el proceso electoral y quedará a consideración del Consejo General, una vez que haya sido admitida por la Secretaría Ejecutiva.

La asunción aplicará cuando se acredite de forma clara la prevalencia de diversos factores sociales que afecten la paz pública y puedan poner a la sociedad de la entidad en cuestión en condiciones de grave riesgo, en donde quede de por medio la organización pacífica de la elección por parte del OPLE correspondiente, debido a la falta de condiciones de equidad o legalidad - principios electorales fundamentales para el desarrollo de la contienda-. De igual forma, dicha atribución podrá ejercerse en situaciones en las que no existan condiciones políticas idóneas de imparcialidad, debido a la intromisión fehaciente de alguno de los poderes públicos de la entidad federativa respectiva, y lo cual implique afectaciones a la organización de la contienda por parte del OPLE.

En el caso de presentarse una petición de asunción, será la Secretaría Ejecutiva del INE la encargada de conducir el proceso, y quien determine su admisión o rechazo - en la situación de que sea notoriamente improcedente-, siendo emplazado el OPLE respectivo a comparecer en el proceso. Cabe destacar que la legislación contempla desechar o suspender el procedimiento de asunción, si la situación que le dio origen se corrige o desaparece.

El Consejo General del INE resolverá si asume o no la organización de las elecciones, antes de que inicie el proceso local respectivo, para lo cual tomará en cuenta las posturas de todos los partidos, actores políticos y poderes del Estado, involucrados en el proceso electoral correspondiente. Para aprobar la resolución de asunción, será necesario el voto a favor de la propuesta de cuando menos ocho consejeros electorales, con la posibilidad de recurrir a la Sala Superior del Tribunal Electoral, como última instancia.

Adicionalmente, existe la posibilidad de que los OPLE soliciten al INE la asunción parcial de alguna actividad correspondiente a la función electoral de dichos organismos, con la diferencia de que la solicitud podrá 
presentarse en cualquier momento del proceso electoral que corresponda, y con efectos únicamente durante el mismo. La solicitud inicial deberá ser aprobada por la mayoría de los integrantes del consejo del Organismo Público Local, y el Instituto resolverá la asunción parcial de algún procedimiento, por mayoría calificada de cuando menos ocho votos de los consejeros electorales.

\section{Atracción}

La atracción queda entendida como una atribución del Instituto Nacional Electoral para atraer a su conocimiento cualquier asunto que se encuentre dentro de la competencia de los OPLE, cuando su trascendencia lo determine o cuando se tenga la intención de sentar un criterio de interpretación para situaciones futuras.

Nuevamente se trata de una atribución que tiene la intención de ser aplicada únicamente en casos excepcionales, y el proceso para ser aplicada es muy similar al de la asunción - es necesario que la petición sea formulada por al menos cuatro consejeros electorales o la mayoría del Consejo General del OPLE en cuestión, y una vez que se cumpla el proceso correspondiente, la facultad de atracción podrá ejercerse si cuenta con al menos ocho votos de los consejeros electorales del INE-.

A diferencia de la asunción, la petición para ejercer la facultad de atracción puede presentarse en cualquier momento, sin importar que el proceso electoral ya haya comenzado, pues tiene la intención de ser aplicada en situaciones trascendentes que puedan afectar o alterar el desarrollo del proceso o la función electoral local. Esta atribución sería aplicada en asuntos novedosos o excepcionales, con el propósito de sentar un criterio de interpretación y fijar un criterio jurídico para situaciones futuras. En todo caso, las decisiones relacionadas con esta facultad del Instituto, podrán ser impugnadas ante el Tribunal Electoral.

\section{Delegación}

Para explicar la tercera forma de interacción entre el INE y los OPLE en la organización de elecciones, me remonto al marco legal de la reciente re- 
forma político electoral. De acuerdo con la Constitución, en el desarrollo de procesos electorales federales y locales, al INE le corresponde:

- Llevar a cabo la capacitación electoral.

- Realizar la geografía electoral, es decir, el diseño y determinación de los distritos electorales y la división del territorio en secciones electorales.

- Llevar el registro del padrón y la lista de electores.

- Ubicar las casillas y designar a los funcionarios de sus mesas directivas.

- Establecer las reglas, lineamientos, criterios y formatos en materia de resultados preliminares; realizar encuestas o sondeos de opinión; llevar a cabo la observación electoral; realizar los conteos rápidos, e imprimir documentos y producir materiales electorales.

- Fiscalizar los ingresos y egresos de los partidos políticos y candidatos.

De acuerdo con la Legipe, la delegación queda entendida como la facultad del INE de delegar a los OPLE las atribuciones recién descritas durante la organización de elecciones en las entidades federativas, con la posibilidad de reasumir su ejercicio directo en el momento que lo considere conveniente. La delegación de funciones es de carácter excepcional y en su caso, se deberán valorar previamente, de forma positiva, las capacidades del OPLE que corresponda, para cumplir adecuadamente las funciones encomendadas.

Para ejercer esta atribución, el Instituto deberá aprobarla con el voto de al menos ocho de sus consejeros electorales. La delegación de funciones se realizará para un OPLE determinado en cada caso, y deberá llevarse a cabo antes de que inicie el proceso electoral correspondiente, pudiendo reasumir las funciones delegadas en cualquier momento, siempre y cuando esta acción sea aprobada nuevamente por la misma mayoría de votos. Una vez finalizado el proceso electoral en cuestión, la delegación quedará sin efectos.

Como se puede notar, estas nuevas atribuciones del INE lo envisten de una jerarquía superior a la de los OPLE, pero una jerarquía que deberá entenderse como de "primero entre pares". Si bien en los casos en que el Instituto decida intervenir en los procesos electorales de los estados, estaría asumiendo funciones que originalmente corresponden a las autoridades electorales de las entidades que correspondan, la legislación es muy clara al establecer que estas medidas son para ser aplicadas en situa- 
ciones extremas que lo ameriten. La nueva autoridad no fue creada con la intención de socavar el desempeño de los organismos locales, sin embargo, es un acierto conferirle la posibilidad de actuar con todo el respaldo legal, en aras de mejorar las prácticas electorales de nuestro país. Por el contrario, la nueva autoridad nacional fue creada como un eje rector que se encargue de apoyar, y en su caso subsidiar, algunas tareas de la organización de los procesos electorales locales. El principio básico para el funcionamiento y eficacia residirá en la colaboración entre la autoridad nacional (el INE) y sus pares en las 32 entidades federativas (los OPLE). 